\title{
Periodic motion in perturbed elliptic oscillators revisited
}

\author{
M. Corbera ${ }^{1} \cdot$ J. Llibre $^{2}$ - C. Valls ${ }^{3}$
}

Received: 22 February 2016 / Accepted: 7 September 2016

(c) Springer Science+Business Media Dordrecht 2016

\begin{abstract}
We analytically study the Hamiltonian system in $\mathbb{R}^{4}$ with Hamiltonian

$H=\frac{1}{2}\left(p_{x}^{2}+p_{y}^{2}\right)+\frac{1}{2}\left(\omega_{1}^{2} x^{2}+\omega_{2}^{2} y^{2}\right)-\varepsilon V(x, y)$

being $V(x, y)=-\left(x^{2} y+a x^{3}\right)$ with $a \in \mathbb{R}$, where $\varepsilon$ is a small parameter and $\omega_{1}$ and $\omega_{2}$ are the unperturbed frequencies of the oscillations along the $x$ and $y$ axis, respectively. Using averaging theory of first and second order we analytically find seven families of periodic solutions in every positive energy level of $H$ when the frequencies are not equal. Four of these seven families are defined for all $a \in \mathbb{R}$ whereas the other three are defined for all $a \neq 0$. Moreover, we provide the shape of all these families of periodic solutions. These Hamiltonians may represent the central parts of deformed galaxies and thus have been extensively used and studied mainly numerically in order to describe local motion in galaxies near an equilibrium point.
\end{abstract}

Keywords Galactic potential $\cdot$ Periodic solutions . Averaging theory

$\triangle$ J. Llibre

jllibre@mat.uab.cat

M. Corbera

montserrat.corbera@uvic.cat

C. Valls

cvalls@math.ist.utl.pt

1 Escola Politècnica Superior, Universitat de Vic-Universitat Central de Catalunya (UVic-UCC), C. de la Laura, 13, 08500 Vic, Catalonia, Spain

2 Departament de Matemàtiques, Universitat Autònoma de Barcelona, 08193 Bellaterra, Barcelona, Catalonia, Spain

3 Departamento de Matemática, Instituto Superior Técnico, Universidade de Lisboa, Av. Rovisco Pais 1049-001, Lisboa, Portugal

\section{Introduction and statement of the main results}

After equilibrium points the periodic solutions are the most simple non-trivial solutions of a differential system. Their study is of special interest because the motion in their neighborhood can be determined by their kind of stability. The stable periodic orbits explain the dynamics of bounded regular motion, while the unstable ones helps to understand the possible chaotic motion of the system. So, periodic orbits play a very important role in understanding the orbital structure of a dynamical system.

Over the last half century dynamical systems perturbing a harmonic oscillator have been used extensively to describe the local motion, i.e. motion near an equilibrium point. The study of this motion have been made mainly using several numerical techniques, see for instance Barbanis (1990), Caranicolas (2001, 2004), Caranicolas and Karanis (1999), Caranicolas and Zotos (2012), Contopoulos (1970b), Elipe et al. (1995), Giorgilli and Galgani (1978), Hénon and Heiles (1964), Karanis and Vozikis (2007), Zotos $(2012 \mathrm{a}, \mathrm{b})$ to cite just a few.

The general form of a potential for a two-dimensional dynamical system composed of two harmonic oscillators with cubic perturbing terms is

$U=\frac{1}{2}\left(\omega_{1}^{2} x^{2}+\omega_{2}^{2} y^{2}\right)+\varepsilon V(x, y)$,

where $\omega_{1}$ and $\omega_{2}$ are the unperturbed frequencies of the oscillator along the $x$ and the $y$ axes, respectively, $\varepsilon$ is the small perturbation parameter and $V$ is the cubic function containing the perturbed terms. We will use the perturbation function

$V(x, y)=-\left(x^{2} y+a x^{3}\right)$, 
with $a \in \mathbb{R}$. These perturbed oscillators are important because they describe the motion of a star under the gravity field of a galaxy, for more information see for instance the paper of Caranicolas (2004) and the references quoted there.

The Hamiltonian associated to the potential $V$ is

$H=H\left(x, y, p_{x}, p_{y}\right)=\frac{1}{2}\left(p_{x}^{2}+p_{y}^{2}\right)+U(x, y)$,

and the corresponding Hamiltonian system is

$\dot{x}=p_{x}$,

$\dot{y}=p_{y}$,

$\dot{p}_{x}=-\omega_{1}^{2} x-\varepsilon \frac{\partial V}{\partial x}$,

$\dot{p}_{y}=-\omega_{2}^{2} y-\varepsilon \frac{\partial V}{\partial y}$.

As usual the dot denotes derivative with respect to the time $t \in \mathbb{R}$. Due to the physical meaning the frequencies $\omega_{1}$ and $\omega_{2}$ are both positive.

We note that system (3) for $\varepsilon=0$ can be solved. It has the solutions on the energy level $H=h$ of the form

$x(t)=C_{1} \cos \left(t \omega_{1}\right)+C_{2} \sin \left(t \omega_{1}\right)$,

$y(t)=C_{3} \cos \left(t \omega_{2}\right)+C_{4} \sin \left(t \omega_{2}\right)$,

$p_{x}(t)=-C_{1} \omega_{1} \sin \left(t \omega_{1}\right)+C_{2} \omega_{1} \cos \left(t \omega_{1}\right)$,

$p_{y}(t)=-C_{3} \omega_{2} \sin \left(t \omega_{2}\right)+C_{4} \omega_{2} \cos \left(t \omega_{2}\right)$,

where $C_{1}, C_{2}, C_{3}, C_{4} \in \mathbb{R}$ satisfy

$h=\frac{1}{2}\left(\omega_{1}^{2}\left(C_{1}^{2}+C_{2}^{2}\right)+\omega_{2}^{2}\left(C_{3}^{2}+C_{4}^{2}\right)\right)$.

Note that the solutions of system (3) for $\varepsilon=0$ given in (4) are periodic if and only if $\omega_{2} / \omega_{1}=p / q$ with $p, q \in \mathbb{N}$ and $p, q$ coprime, where as usual $\mathbb{N}$ denotes the set of positive integers. The period of these periodic solutions is

$T=\frac{2 p \pi}{\omega_{2}}=\frac{2 q \pi}{\omega_{1}}$.

As far as we know there are no rigorous analytic studies of the existence of periodic solutions for the Hamiltonian system (3) with $\omega_{1} \neq \omega_{2}$. Periodic orbits when $a=0$ have been studied by several authors from both analytical and numerical point of view by using different techniques, see for instance Contopoulos and Moutsoulas (1965), Contopoulos and Zikides (1980), Davoust (1983), Caranicolas and Innanen (1992) for $\omega_{1}=\omega_{2}$, or Contopoulos (1970a) for a numerical study for some values $\omega_{1} \neq \omega_{2}$. The perturbed potential with $\omega_{2}=\omega_{1}$ and $a \neq 0$ has been studied analytically in Elipe et al. (1995), where the authors found six families of periodic orbits by using similar techniques than the ones in Miller (1991).
In this paper we will study the periodic orbits of the Hamiltonian system (3) with perturbed potential (1) by using averaging theory. More precisely, we will study the cases $\omega_{2}=2 \omega_{1}$ and $\omega_{2}=3 \omega_{1}$ with first and second order averaging, respectively. These cases together with the case $\omega_{2}=\omega_{1}$ are the unique cases that we are able to study with these averaging techniques. We will prove the existence of families of periodic solutions parameterized by the energy in every energy level $H=h>0$, and these families will be given explicitly up to first order in the small parameter $\varepsilon$. The case $a=0$ has been studied by several authors so it is not considered in this work. See also Alfaro et al. (2013) for other uses of averaging theory.

Theorem 1 The following statements hold for the Hamiltonian system (3) with Hamiltonian $H$ given in (2) and $V(x, y)$ in (1).

(a) Using averaging theory of first order for $|\varepsilon| \neq 0$ sufficiently small at every positive energy level $H=h$ and with $\omega_{2}=2 \omega_{1}>0$, we find for the Hamiltonian system (3), four periodic solutions (two linearly stable and two unstable) bifurcating from the periodic solutions of (4) with a period tending to $2 \pi / \omega_{1}$ as $\varepsilon \rightarrow 0$. The two unstable periodic orbits have a stable and an unstable manifold, each one formed by two cylinders. All these periodic solutions can be written as $\left(\tilde{x}(t), \tilde{y}(t), \tilde{p}_{x}(t), \tilde{p}_{y}(t)\right)+O(\varepsilon)$ with $\left(\tilde{x}(t), \tilde{y}(t), \tilde{p}_{x}(t), \tilde{p}_{y}(t)\right)$ being respectively,

$$
\begin{aligned}
& \left(0, \pm \frac{\sqrt{h}}{\sqrt{2} \omega_{1}} s_{2}, 0, \pm \sqrt{2 h} c_{2}\right), \\
& \left(\frac{2 \sqrt{h}}{\sqrt{3} \omega_{1}} c_{1}, \pm \frac{\sqrt{h}}{\sqrt{6} \omega_{1}} c_{2},-\frac{2 \sqrt{h}}{\sqrt{3}} s_{1}, \mp \sqrt{\frac{2 h}{3}} s_{2}\right),
\end{aligned}
$$

where $c_{1}=\cos \tau, c_{2}=\cos (2 \tau), s_{1}=\sin \tau s_{2}=\sin (2 \tau)$ and $\tau=\omega_{1} t$.

(b) Using averaging theory of second order for $|\varepsilon| \neq 0$ sufficiently small at every positive energy level $H=$ $h$ and with $\omega_{2}=3 \omega_{1}>0$, for each $a \neq 0$ we find for the Hamiltonian system (3), three periodic solutions (two linearly stable and one unstable) bifurcating from the periodic solutions of (4) with a period tending to $2 \pi / \omega_{1}$ as $\varepsilon \rightarrow 0$. The unstable periodic orbit has a stable and an unstable manifold, each one formed by two cylinders. All these periodic solutions can be written as $\left(\tilde{x}(t), \tilde{y}(t), \tilde{p}_{x}(t), \tilde{p}_{y}(t)\right)+O(\varepsilon)$ with $\left(\tilde{x}(t), \tilde{y}(t), \tilde{p}_{x}(t), \tilde{p}_{y}(t)\right)$ being respectively,

$$
\left(\frac{r_{i}}{\omega_{1}} c_{1}, \mp \frac{\sqrt{2 h-r_{i}^{2}}}{3 \omega_{1}} c_{3},-r_{i} s_{1}, \pm \sqrt{2 h-r_{i}^{2}} s_{3}\right)
$$

for $i=1,2,3$, where $c_{1}=\cos \tau, c_{3}=\cos (3 \tau), s_{1}=$ $\sin \tau, s_{3}=\sin (3 \tau), \tau=\omega_{1} t$ and $r_{1}, r_{2}, r_{3}$ as well as 
the stability of the solutions for each $r_{i}$ are given in the proof of the theorem.

The proof of Theorem 1 is given in Sect. 3 .

We note that the two solutions

$\left(x(t), y(t), p_{x}(t), p_{y}(t)\right)=\left(0, \pm \frac{\sqrt{h}}{\sqrt{2} \omega_{1}} s_{2}, 0, \pm \sqrt{2 h} c_{2}\right)$,

are explicit periodic solutions of our Hamiltonian system for all $\varepsilon$. Moreover they are axial periodic solutions living on the $y$-axis. While the other five periodic solutions described in Theorem 1 are non-axial periodic solutions.

The origin of the Hamiltonian system (3) with the potential (1) is always an equilibrium point. The periodic orbits given in Theorem 1 are near the origin for small values of the energy $h>0$. Near the stable periodic orbits of Theorem 1 the KAM 2-dimensional tori will persist for small values of $\varepsilon$, provided that the Kolmogorov condition or the isoenergetic non-degeneracy condition holds. These tori can be studied following the arguments given in the papers of Cushman et al. (2007) and Belmonte et al. (2007).

We must mention that there are other analytical results, which do not use the averaging method, for studying the periodic orbits with some resonances $\omega_{1}: \omega_{2}$ for Hamiltonian systems with Hamiltonians of the form

$H=\frac{1}{2}\left(p_{x}^{2}+p_{y}^{2}\right)+\frac{1}{2}\left(\omega_{1} x^{2}+\omega_{2} y^{2}\right)+\varepsilon W(x, y)$,

where $\varepsilon$ is a small parameter. Thus, for instance in Cushman et al. (2007) the authors studied the periodic orbits with resonance $1: \pm 2$ using the Birkhoff normal form. In Marchesiello and Pucacco (2013) the authors studied the periodic orbits with resonance $1: 2$ using the Lie transform normal form theory. In Pucacco and Marchesiello (2014) the periodic orbits with resonance $1: 1$ and symmetry $\mathbb{Z}_{2} \times \mathbb{Z}_{2}$ are studied using normal form theory. In Schmidt and Dullin (2010) some properties of the periodic orbits with resonance $p: \pm q$ are studied using the Birkhoff normal form. We remark that the potentials $W(x, y)$ of those papers are different between them, and different from the potential studied in this paper. More references about these normal form techniques for studying the periodic orbits of Hamiltonian systems can be found in the references quoted in those four mentioned papers.

In Sect. 2 we present a summary of the results on the averaging theory that we shall need for proving our results.

\section{The averaging theory of first and second order}

In this section we summarize the averaging theory of second order, it provides sufficient conditions for the existence of periodic solutions for a periodic differential system depending on a small parameter. See Buică and Llibre (2004) for additional details and for the proofs of the results stated in this section.

Theorem 2 Consider the differential system

$\dot{x}(t)=\varepsilon F_{1}(t, x)+\varepsilon^{2} F_{2}(t, x)+\varepsilon^{3} R(t, x, \varepsilon)$,

where $F_{1}, F_{2}: \mathbb{R} \times D \rightarrow \mathbb{R}^{n}, R: \mathbb{R} \times D \times\left(-\varepsilon_{f}, \varepsilon_{f}\right) \rightarrow \mathbb{R}^{n}$ are continuous and T-periodic functions in the first variable, and $D$ is an open subset of $\mathbb{R}^{n}$. Assume that the following hypotheses hold.

(i) $F_{1}(t, \cdot) \in C^{1}(D)$ for all $t \in \mathbb{R}, F_{1}, F_{2}, R$ and $D_{x} F_{1}$ are locally Lipschitz with respect to $x$, and $R$ is differentiable with respect to $\varepsilon$. We define $f_{1}, f_{2}: D \rightarrow \mathbb{R}^{n}$ as

$$
\begin{aligned}
& f_{1}(z)=\int_{0}^{T} F_{1}(s, z) d s \\
& f_{2}(z)=\int_{0}^{T}\left[D_{z} F_{1}(s, z) y_{1}(s, z)+F_{2}(s, z)\right] d s .
\end{aligned}
$$

where

$$
y_{1}(s, z)=\int_{0}^{s} F_{1}(t, z) d t .
$$

(ii) For $V \subset D$ an open and bounded set and for each $\varepsilon \in$ $\left(-\varepsilon_{f}, \varepsilon_{f}\right) \backslash\{0\}$, there exist $a \in V$ such that

(ii.1) if $f_{1}(z) \neq \equiv$, then $f_{1}(a)=0$ and $d_{B}\left(f_{1}, a\right) \neq 0$, where $d_{B}\left(f_{1}, a\right)$ denotes the Brouwer degree of the function $f_{1}: V \rightarrow \mathbb{R}^{n}$ at the fixed point $a$; and

(ii.2) if $f_{1}(z) \equiv 0$ and $f_{2}(z) \neq \equiv$, then $f_{2}(a)=0$ and $d_{B}\left(f_{2}, a\right) \neq 0$.

Then for $|\varepsilon|>0$ sufficiently small, there exists a T-periodic solution $\varphi(t, \varepsilon)$ of the system such that $\varphi(0, \varepsilon) \rightarrow a$ when $\varepsilon \rightarrow 0$. The kind of stability or instability of the limit cycle $\varphi(t, \varepsilon)$ is given by the eigenvalues of the Jacobian matrix $\left.D_{z}\left(f_{1}(z)+\varepsilon f_{2}(z)\right)\right|_{z=a}$.

Note that a sufficient condition for showing that the Brouwer degree of a function $f$ at a fixed point $a$ is nonzero, is that the Jacobian of the function $f$ at $a$ (when it is defined) is non-zero, see Lloyd (1978).

Under the assumption (ii.1) Theorem 2 provides the $a v$ eraging theory of first order, and it provides the averaging theory of second order when assumption (ii.2) holds.

\section{Proof of Theorem 1}

For proving Theorem 1 we shall use Theorem 2. The first step is to write system (3) in such a way that conditions of 
Theorem 2 be satisfied. We write system (3) and the Hamiltonian (2) in polar coordinates

$x=\frac{r \cos \theta}{\omega_{1}}, \quad p_{x}=r \sin \theta$,

$y=\frac{\rho \cos \varphi}{\omega_{2}}, \quad p_{y}=\rho \sin \varphi$

where $\varphi=\alpha+\omega_{2} \theta / \omega_{1}$ and we get the system of equations

$\dot{r}=\frac{\varepsilon r \sin (2 \theta)}{2 \omega_{1}^{2} \omega_{2}}\left(3 a r \omega_{2} \cos \theta+2 \rho \omega_{1} \cos \varphi\right)$,

$\dot{\theta}=-\omega_{1}+\frac{\varepsilon \cos ^{2} \theta}{\omega_{1}^{2} \omega_{2}}\left(3 \operatorname{ar} \omega_{2} \cos \theta+2 \rho \omega_{1} \cos \varphi\right)$,

$\dot{\rho}=\frac{\varepsilon r^{2} \cos ^{2} \theta}{\omega_{1}^{2}} \sin \varphi$,

$\dot{\alpha}=\frac{\varepsilon \cos ^{2} \theta}{\rho \omega_{1}^{3}}\left(\omega_{1}\left(r^{2}-2 \rho^{2}\right) \cos \varphi-3 a \rho r \omega_{2} \cos \theta\right)$,

and the Hamiltonian

$H=\frac{1}{2}\left(r^{2}+\rho^{2}\right)-\frac{\varepsilon}{\omega_{1}^{2}} r^{2} \cos ^{2} \theta\left(\frac{\operatorname{arcos} \theta}{\omega_{1}}+\frac{\rho}{\omega_{2}} \cos \varphi\right)$.

We note that system (10) is periodic in the variable $\theta$ if and only if $\omega_{2}=p \omega_{1} / q$ for some $p, q \in \mathbb{N}$ coprime. Moreover its period is $2 q \pi$. We write system (10) by taking as the new independent variable the angular variable $\theta$ and we obtain

$r^{\prime}=\frac{\dot{r}}{\dot{\theta}}, \quad \rho^{\prime}=\frac{\dot{\rho}}{\dot{\theta}}, \quad \alpha^{\prime}=\frac{\dot{\alpha}}{\dot{\theta}}$

where the prime denotes derivative with respect to $\theta$. We compute $\rho=\rho_{0}+\rho_{1} \varepsilon+O\left(\varepsilon^{2}\right)$ by solving equation $H=h$ and we get

$$
\begin{aligned}
& \rho_{0}=\sqrt{2 h-r^{2}}, \\
& \rho_{1}=\frac{r^{2} \cos ^{2} \theta}{\omega_{1}^{3}}\left(\frac{\operatorname{arcos} \theta}{\sqrt{2 h-r^{2}}}+\frac{\omega_{1}}{\omega_{2}} \cos \varphi\right) .
\end{aligned}
$$

We substitute the expression of $\rho$ into (12) and we develop the resulting equations in power series of $\varepsilon$ up to second order. In the energy level $H=h>0$ the equations of motion become

$$
\begin{aligned}
& r^{\prime}=\varepsilon F_{11}+\varepsilon^{2} F_{21}+O\left(\varepsilon^{3}\right), \\
& \alpha^{\prime}=\varepsilon F_{12}+\varepsilon^{2} F_{22}+O\left(\varepsilon^{3}\right),
\end{aligned}
$$

with

$$
\begin{aligned}
F_{11}= & -\frac{r \sin (2 \theta)}{2 \omega_{1}^{3} \omega_{2}}\left(3 \operatorname{ar} \omega_{2} \cos \theta\right. \\
& \left.+2 \omega_{1} \sqrt{2 h-r^{2}} \cos \varphi\right), \\
F_{12}= & \frac{\cos ^{2} \theta}{\omega_{1}^{4} \sqrt{2 h-r^{2}}}\left(3 a r \omega_{2} \sqrt{2 h-r^{2}} \cos \theta\right. \\
& \left.+\omega_{1}\left(4 h-3 r^{2}\right) \cos \varphi\right), \\
F_{21}= & \frac{r \sin \theta \cos ^{3} \theta}{\omega_{1}^{6} \omega_{2}^{2} \sqrt{2 h-r^{2}}}\left(-9 a^{2} r^{2} \omega_{2}^{2} \sqrt{2 h-r^{2}} \cos ^{2} \theta\right. \\
& +2 a r \omega_{1} \omega_{2}\left(5 r^{2}-12 h\right) \cos \theta \cos \varphi \\
& \left.+2 \omega_{1}^{2} \sqrt{2 h-r^{2}}\left(r^{2}-4 h\right) \cos ^{2} \varphi\right), \\
F_{22}= & \frac{\cos ^{4} \theta}{\omega_{1}^{7} \omega_{2}\left(2 h-r^{2}\right)^{3 / 2}}\left(9 a^{2} r^{2} \omega_{2}^{2}\left(2 h-r^{2}\right)^{3 / 2} \cos ^{2} \theta\right. \\
& +2 a r \omega_{1} \omega_{2}\left(24 h^{2}-25 h r^{2}+7 r^{4}\right) \cos ^{2} \theta \cos ^{2} \varphi \\
& \left.+\omega_{1}^{2} \sqrt{2 h-r^{2}}\left(16 h^{2}-16 h r^{2}+5 r^{4}\right) \cos ^{2} \varphi\right) .
\end{aligned}
$$

In order that the differential system (14) be in the normal form (7) for applying the averaging theory we need that this system be periodic in the variable $\theta$. This implies that $\omega_{2} / \omega_{1}$ be rational. So from now on we assume that $\omega_{2}=p \omega_{1} / q$ with $p, q \in \mathbb{N}$, so system (12) is $2 q \pi$-periodic in the variable $\theta$. Then system (14) is in the normal form (7) for applying the averaging theory with $T=2 q \pi, \mathbf{x}=$ $(r, \alpha), t=\theta, F_{1}(\theta, \mathbf{x})=\left(F_{11}, F_{12}\right), F_{2}(\theta, \mathbf{x})=\left(F_{21}, F_{22}\right)$ and $\varepsilon^{2} R(\theta, \mathbf{x}, \varepsilon)$ is $O\left(\varepsilon^{3}\right)$. We also observe that $F$ and $R$ are $\mathcal{C}^{2}$ in $\mathbf{x}$ and $2 q \pi$-periodic in $\theta$ in an open set not containing $r=\sqrt{2 h}$. After some computations, from (8) we get

$$
f_{1}(\mathbf{x})=\int_{0}^{2 q \pi} F_{1}(\theta, \mathbf{x}) d \theta=\left(f_{11}(\mathbf{x}), f_{12}(\mathbf{x})\right),
$$

with

$$
\begin{aligned}
& f_{11}(\mathbf{x})=\int_{0}^{2 q \pi} F_{11} d \theta= \begin{cases}0 & p \neq 2 q, \\
\tilde{f}_{11}(r, \alpha) & p=2 q,\end{cases} \\
& f_{12}(\mathbf{x})=\int_{0}^{2 q \pi} F_{12} d \theta= \begin{cases}0 & p \neq 2 q, \\
\tilde{f}_{12}(r, \alpha) & p=2 q,\end{cases}
\end{aligned}
$$

where

$$
\begin{aligned}
& \tilde{f}_{11}(r, \alpha)=\frac{\pi q r \sqrt{2 h-r^{2}} \sin \alpha}{2 \omega_{1}^{3}}, \\
& \tilde{f}_{12}(r, \alpha)=\frac{\pi q\left(4 h-3 r^{2}\right) \cos \alpha}{2 \omega_{1}^{3} \sqrt{2 h-r^{2}}} .
\end{aligned}
$$


Case 1: $p=2 q$. Since $p$ and $q$ are coprime, we take $q=1$. The solutions of system $f_{1}(\mathbf{x})=\left(f_{11}(\mathbf{x}), f_{12}(\mathbf{x})\right)=0$ are $\left(r_{(1, j)}, \alpha_{(1, j)}\right)=(0, \pi / 2+j \pi)$ and $\left(r_{(2, j)}, \alpha_{(2, j)}\right)=$ $(2 \sqrt{h / 3}, j \pi)$ with $j=0,1$.

Now we compute the Jacobian matrix of $f_{1}$ and we get

$$
\begin{aligned}
\mathcal{J} & =D_{\mathbf{x}} f_{1}=\left(\begin{array}{ll}
\frac{\partial f_{11}}{\partial r} & \frac{\partial f_{11}}{\partial \alpha} \\
\frac{\partial f_{12}}{\partial r} & \frac{\partial f_{12}}{\partial \alpha}
\end{array}\right) \\
& =\pi\left(\begin{array}{cc}
\frac{\left(h-r^{2}\right) \sin \alpha}{\sqrt{2 h-r^{2}} \omega_{1}^{3}} & \frac{r \sqrt{2 h-r^{2}} \cos \alpha}{2 \omega_{1}^{3}} \\
\frac{r\left(3 r^{2}-8 h\right) \cos \alpha}{2\left(2 h-r^{2}\right)^{3 / 2} \omega_{1}^{3}} & -\frac{\left(4 h-3 r^{2}\right) \sin \alpha}{2 \sqrt{2 h-r^{2}} \omega_{1}^{3}}
\end{array}\right) .
\end{aligned}
$$

By evaluating the determinant of $\mathcal{J}$ on the solutions $\left(r_{(i, j)}\right.$, $\left.\alpha_{(i, j)}\right)$ for $i=1,2$ and $j=0,1$ we get

$\operatorname{det}(\mathcal{J})_{(r, \alpha)=\left(r_{(1, j)}, \alpha_{(1, j)}\right)}=-\frac{\pi^{2} h}{\omega_{1}^{6}} \neq 0$,

$\operatorname{det}(\mathcal{J})_{(r, \alpha)=\left(r_{(2, j)}, \alpha_{(2, j)}\right)}=\frac{2 \pi^{2} h}{\omega_{1}^{6}} \neq 0$.

If follows from Theorem 2 that for any given $h>0$ and for $|\varepsilon|$ sufficiently small, system (14) has the four $2 \pi$-periodic solutions that are $\left(r^{(i, j)}(\theta, \varepsilon), \alpha^{(i, j)}(\theta, \varepsilon)\right)$ for $i=1,2$ and $j=0,1$ such that the solution $\left(r^{(i, j)}(\theta, \varepsilon), \alpha^{(i, j)}(\theta, \varepsilon)\right)$ tends to $\left(r_{(i, j)}, \alpha_{(i, j)}\right)$ when $\varepsilon \rightarrow 0$.

The eigenvalues of the matrix $\mathcal{J}$ evaluated at the solution $(r, \alpha)=\left(r_{(1, j)}, \alpha_{(1, j)}\right)$ for $j=0,1$ are

$\lambda_{1}=-(-1)^{j} \frac{\sqrt{2 h} \pi}{\omega_{1}^{3}}, \quad \lambda_{2}=(-1)^{j} \frac{\pi \sqrt{h}}{\sqrt{2} \omega_{1}^{3}}$,

and the eigenvalues of the matrix $\mathcal{J}$ evaluated at the solution $(r, \alpha)=\left(r_{(2, j)}, \alpha_{(2, j)}\right)$ for $j=0,1$ are

$\lambda_{1,2}= \pm \frac{i \sqrt{2 h} \pi}{\omega_{1}^{3}}$,

where $i=\sqrt{-1}$. So the periodic solutions $\left(r^{(i, j)}(\theta, \varepsilon)\right.$, $\alpha^{(i, j)}(\theta, \varepsilon)$ ) for $j=0,1$ are linearly unstable when $i=1$ and linearly stable when $i=2$. Since the eigenvalues of the matrix Jacobian matrix $\mathcal{J}$ evaluated at $\left(r^{(i, j)}(\theta, \varepsilon)\right.$, $\left.\alpha^{(i, j)}(\theta, \varepsilon)\right)$ provide the stability of the fixed point corresponding to the Poincaré map defined in a neighborhood of the periodic solution associated to $\left(r^{(i, j)}(\theta, \varepsilon), \alpha^{(i, j)}(\theta, \varepsilon)\right)$ (see for instance the proof of Theorem 11.6 of Verhulst 1991), and this fixed point is locally a saddle for $i=1$, we obtain that the unstable orbits have a stable and an unstable manifolds formed by two cylinders.

Now we shall go back through the changes of variables in order to see how the $2 \pi$-periodic solutions $\left(r^{(i, j)}(\theta, \varepsilon)\right.$, $\alpha^{(i, j)}(\theta, \varepsilon)$ ), with $i=1,2$ and $j=0,1$ of the differential system (14) looks in the initial Hamiltonian system (3). By substituting $\left(r^{(i, j)}(\theta, \varepsilon), \alpha^{(i, j)}(\theta, \varepsilon)\right)$ into equation $H=h$ with $H$ given in (11) we get $\rho^{(i, j)}(\theta, \varepsilon)$. Then

$\left(r^{(i, j)}(\theta, \varepsilon), \rho^{(i, j)}(\theta, \varepsilon), \alpha^{(i, j)}(\theta, \varepsilon)\right)$,

is a $2 \pi$-periodic solution for the differential system (12). This solution provides the $2 \pi / \omega_{1}$-periodic solution for the differential system (10)

$$
\begin{aligned}
& \left(r^{(i, j)}\left(\sigma^{(i, j)}, \varepsilon\right), \sigma^{(i, j)}, \rho^{(i, j)}\left(\sigma^{(i, j)}, \varepsilon\right), \alpha^{(i, j)}\left(\sigma^{(i, j)}, \varepsilon\right)\right) \\
& =\left(r_{(i, j)},-\omega_{1} t, \sqrt{2 h-r_{(i, j)}^{2}}, \alpha_{(i, j)}\right)+O(\varepsilon) .
\end{aligned}
$$

Here $\sigma^{(i, j)}=\theta^{(i, j)}(t, \varepsilon)$. Going back to the change of variables (3) we get the four $2 \pi / \omega_{1}$ periodic solutions of system (3) given in (5), which clearly are different solutions. This completes the proof of statement (a) of Theorem 2.

Case 2: $p \neq 2 q$. Since $f_{11}(\mathbf{x})=0$ and $f_{12}(\mathbf{x})=0$, we need to consider averaging of second order. After tedious computations, from (9), we get $f_{2}(\mathbf{x})=\left(f_{21}(r, \alpha), f_{22}(r, \alpha)\right)$ where

$f_{21}(r, \alpha)= \begin{cases}0 & p \neq q, \quad p \neq 3 q, \quad p \neq 5 q, \\ 0 & p=5 q, \\ g_{0}(r, \alpha) & p=q, \\ g_{1}(r, \alpha) & p=3 q,\end{cases}$

and

$f_{22}(r, \alpha)= \begin{cases}g_{2}(r, \alpha) & p \neq q, p \neq 3 q, \quad p \neq 5 q, \\ g_{3}(r, \alpha) & p=5 q \\ g_{4}(r, \alpha) & p=q \\ g_{5}(r, \alpha) & p=3 q\end{cases}$

where

$$
\begin{aligned}
g_{0}(r, \alpha)= & \frac{\pi r q \sin \alpha}{2 \omega_{1}^{6}}\left(5 a r \sqrt{2 h-r^{2}}\right. \\
& \left.+\cos \alpha\left(8 h-4 r^{2}\right)\right), \\
g_{1}(r, \alpha)= & -\frac{\pi a q r^{2} \sqrt{2 h-r^{2}} \sin \alpha}{2 \omega_{1}^{6}}, \\
g_{2}(r, \alpha)= & \frac{\pi\left(3 p^{2} r^{2}\left(5 a^{2} p^{2}-20 a^{2} q^{2}+q^{2}\right)-8 h q^{4}\right)}{2 p \omega_{1}^{6}\left(p^{2}-4 q^{2}\right)}, \\
g_{3}(r, \alpha)= & \frac{\pi q}{210 \omega_{1}^{6}}\left(75\left(105 a^{2}+1\right) r^{2}-8 h\right), \\
g_{4}(r, \alpha)= & \frac{\pi q}{\omega_{1}^{6}}\left(\frac{1}{2}\left(15 a^{2}-1\right) r^{2}+2 \cos (2 \alpha)\left(h-r^{2}\right)\right. \\
& \left.+\frac{8}{6} h+\frac{5 a r \cos \alpha\left(3 h-2 r^{2}\right)}{\sqrt{2 h-r^{2}}}\right),
\end{aligned}
$$




$$
\begin{aligned}
g_{5}(r, \alpha)= & -\frac{\pi q}{30 \omega_{1}^{6}}\left(8 h-27\left(25 a^{2}+1\right) r^{2}\right. \\
& \left.+\frac{30 \operatorname{ar}\left(3 h-2 r^{2}\right) \cos \alpha}{\sqrt{2 h-r^{2}}}\right) .
\end{aligned}
$$

Case 2.1: $p \neq q, p \neq 3 q$ and $p \neq 5 q$. There are no $a \in \mathbb{R}$ and $p, q \in \mathbb{N}$ such that $f_{22}(r, \alpha)$ be identically 0 . Since the function $f_{21}$ is identically 0 and $f_{22}$ is not identically 0 , the averaging theory does not provide any information on the periodic solutions of (14) in this case.

Case 2.2: $p=5 q$. As in the previous case the averaging theory does not provide any information on the periodic solutions of (14).

Case 2.2: $p=q$. This case corresponds to the case $\omega_{1}=\omega_{2}$ studied in Elipe et al. (1995). So it is not considered in this work.

Case 2.3: $p=3 q$. Since $p$ and $q$ are coprime, we take $q=1$. We seek solutions of system $f_{2}(\mathbf{x})=0$ for which the Jacobian of $f_{2}$ evaluated at the solution be non-zero. The solutions of equation $f_{21}(r, \alpha)=0$ are $r=0, r=\sqrt{2 h}$ and $\alpha=j \pi$ for $j=0,1$. The solution $r=0$ does not provide solutions of $f_{2}(\mathbf{x})=0$, because $f_{22}(0, \alpha)=-(4 h \pi) /$ $\left(15 \omega_{1}^{6}\right) \neq 0$. The solution $r=\sqrt{2 h}$ is not valid because in this case system $f_{2}(\mathbf{x})=0$ is not defined. Now we analyze the solutions $\alpha=j \pi$. For this, we need an auxiliary result.

Lemma 3 Assume $h>0$ and let $t_{1}(a, h), t_{2}(a, h), t_{3}(a, h)$ be the three real solutions of the polynomial

$$
\begin{aligned}
& \left(455625 a^{4}+40050 a^{2}+729\right) t^{3} \\
& -\left(911250 a^{4} h+94500 a^{2} h+1890 h\right) t^{2} \\
& +\left(29700 a^{2} h^{2}+928 h^{2}\right) t-128 h^{3}=0,
\end{aligned}
$$

ordered from big to small. Then the following statements hold for the equation $f_{22}(r, j \pi)=0$.

(a) For $a>0$ it has a unique positive real solution $r=$ $\sqrt{t_{2}(a, h)}$ when $j=0$, and two positive real solutions $r=\sqrt{t_{1}(a, h)}$ and $r=\sqrt{t_{3}(a, h)}$ when $j=1$.

(b) For $a<0$ it has two positive real solutions $r=$ $\sqrt{t_{1}(a, h)}$ and $r=\sqrt{t_{3}(a, h)}$ when $j=0$, and one positive real solution $r=\sqrt{t_{2}(a, h)}$ when $j=1$.

(c) The Jacobian matrix of $f_{2}$ evaluated at $(r, \alpha)=(r(a$, $h), j \pi)$, where $r(a, h)$ is any solution of equation $f_{22}(r, j \pi)=0$ with $a \neq 0$ and $j=0,1$, is different from zero.

Proof If $r \neq \sqrt{2 h}$, equation $f_{22}(r, j \pi)=0$ is equivalent to equation

$$
\left(8 h-27\left(25 a^{2}+1\right) r^{2}\right) \sqrt{2 h-r^{2}}=-30 a r\left(3 h-2 r^{2}\right)(-1)^{j} .
$$

Squaring both sides of (15) we get the polynomial equation (independent of $j$ )

$$
\begin{aligned}
& \left(455625 a^{4}+40050 a^{2}+729\right) r^{6} \\
& -\left(911250 a^{4} h+94500 a^{2} h+1890 h\right) r^{4} \\
& +\left(29700 a^{2} h^{2}+928 h^{2}\right) r^{2}-128 h^{3}=0 .
\end{aligned}
$$

Equation (16) has the solutions of (15) and probably new ones.

By doing the change of variables $t=r^{2}$ in (16) we obtain a new cubic polynomial equation $\bar{g}(t)=0$ with positive discriminant

$$
\begin{aligned}
& a^{2}\left(405 a^{2}+13\right)^{2}\left(5843390625 a^{6}+604158750 a^{4}\right. \\
& \left.\quad+19231425 a^{2}+194672\right) h^{6},
\end{aligned}
$$

unless a positive real constant. So the polynomial $\bar{g}(t)$ when $a \neq 0$ has three real roots, for more details about the discriminant of a cubic polynomial see Abramowitz and Stegun (1972). Using the Descartes rule on the signs of a polynomial we can see that these roots cannot be negative, and of course they are non-zero, so they are positive and we denote them as $t_{1}(a, h)>t_{2}(a, h)>t_{3}(a, h)$ for $a \neq 0$ and $h>0$. When $a=0$ the polynomial $\bar{g}(t)$ has two roots $t=8 h / 27$ and $t=2 h$. Notice that the last root does not provide solutions of equation $f_{22}(r, j \pi)=0$ because $r= \pm \sqrt{2 h}$.

Now we study which of these solutions provide solutions of equation $f_{22}(r, j \pi)=0$. It is not difficult to check that the factors $K_{1}=\left(3 h-2 r^{2}\right)$ and $K_{2}=\left(8 h-27\left(25 a^{2}+1\right) r^{2}\right)$ in $f_{22}(r, j \pi)$ do not change their sing on the solutions $r=$ $\pm \sqrt{t_{i}(a, h)}$ for all $i=1,2,3$. In particular,

$$
\begin{cases}K_{1}, K_{2}<0 & \text { on } r= \pm \sqrt{t_{1}(a, h)}, \\ K_{1}>0, K_{2}<0 & \text { on } r= \pm \sqrt{t_{2}(a, h)} \\ K_{1}, K_{2}>0 & \text { on } r= \pm \sqrt{t_{3}(a, h)}\end{cases}
$$

Analyzing the signs of the two summands of $f_{22}(r, j \pi)$ we conclude that $f_{22}(r, j \pi)=0$ has the solutions: $r=$ $-\sqrt{t_{1}(a, h)}, r=\sqrt{t_{2}(a, h)}$ and $r=-\sqrt{t_{3}(a, h)}$ when either $j=0$ and $a>0$ or $j=\pi$ and $a<0$; and $r=\sqrt{t_{1}(a, h)}$, $r=-\sqrt{t_{2}(a, h)}$ and $r=\sqrt{t_{3}(a, h)}$ when either $j=1$ and $a>0$ or $j=0$ and $a<0$. This proves statements (a) and (b).

To prove statement (c) we seek for the solutions $r=$ $r(a, h)$ of the equation $f_{22}(r, j \pi)=0$ such that the Jacobian of $f_{2}$ evaluated at $(r, \alpha)=(r(a, h), j \pi)$ is equal to zero. The Jacobian of $f_{2}$ evaluated at $\alpha=j \pi$ is

$$
\begin{aligned}
\operatorname{det}(\mathcal{J})= & \frac{\pi^{2} a^{2} r^{2}\left(3 h^{2}-6 h r^{2}+2 r^{4}\right)}{\omega_{1}^{12}\left(r^{2}-2 h\right)} \\
& +\frac{9 \pi^{2} a\left(25 a^{2}+1\right)(-1)^{j} r^{3} \sqrt{2 h-r^{2}}}{10 \omega_{1}^{12}} .
\end{aligned}
$$


We transform equation $\operatorname{det}(\mathcal{J})=0$ into a polynomial equation in the variable $t=r^{2}$ as we have done with equation $f_{22}(r, j \pi)=0$ and we get

$$
\begin{aligned}
\bar{g}_{1}(t)= & \pi^{4} a^{2} t^{2}\left[\left(-50625 a^{4}-4450 a^{2}-81\right) t^{4}\right. \\
& +6\left(50625 a^{4}+4450 a^{2}+81\right) h t^{3} \\
& -12\left(50625 a^{4}+4450 a^{2}+81\right) h^{2} t^{2} \\
& \left.+72\left(5625 a^{4}+500 a^{2}+9\right) h^{3} t-900 a^{2} h^{4}\right] .
\end{aligned}
$$

We compute the resultant between the polynomial $\bar{g}(t)$ and $\bar{g}_{1}(t)$ with respect to the variable $t$ and we obtain a polynomial $P(a, h)$, in the variables $a$ and $h$, with the property that if the polynomials $\bar{g}(t)$ and $\bar{g}_{1}(t)$ have a common root, this occurs for values of $(a, h)$ such that $P(a, h)=0$, for more information about the resultant of two polynomials see for instance Lang (1993), Olver (1999). The polynomial $P(a, h)$ can be factorized as

$-26214400 \pi^{12} a^{8} h^{18} P_{1}(a) P_{2}(a) P_{3}(a)$,

where $P_{1}(a), P_{2}(a)$, and $P_{3}(a)$ are polynomials in the variable $a$ of degrees 4,6 , and 10 respectively whose coefficients are all positive. Since $P(a, h)$ is zero if and only if $a=0$ (recall that $h>0)$, there are no solutions of system $\bar{g}(t)=0$, $\bar{g}_{1}(t)=0$ with $a \neq 0$, and consequently there are no solutions of system $f_{2}(\mathbf{x})=0$ with $a \neq 0$ having Jacobian equal to zero. On the other hand it is easy to check that the solution $f_{22}(r, j \pi)=0$ for $a=0, r=\frac{2}{3} \sqrt{\frac{2}{3}} \sqrt{h}$ has Jacobian equal to zero. This completes the proof of the lemma.

Let $r_{i}=\sqrt{t_{i}(a, h)}$ for $i=1,2,3$; and let $\mathbf{x}_{1}=\left(r_{2}, 0\right)$, $\mathbf{x}_{2}=\left(r_{1}, \pi\right), \mathbf{x}_{3}=\left(r_{3}, \pi\right), \mathbf{x}_{4}=\left(r_{1}, 0\right), \mathbf{x}_{5}=\left(r_{3}, 0\right)$, and $\mathbf{x}_{6}=\left(r_{2}, \pi\right)$. From Lemma 3 together with Theorem 2, for any given $h>0$ and for $|\varepsilon|$ sufficiently small, system (14) when $a>0$ has three $2 \pi$-periodic solutions $\left(r^{i}(\theta, \varepsilon), \alpha^{i}(\theta, \varepsilon)\right)$ with $i=1,2,3$, such that $\left(r^{i}(\theta, \varepsilon)\right.$, $\left.\alpha^{i}(\theta, \varepsilon)\right)$ tends to $\mathbf{x}_{i}$ when $\varepsilon \rightarrow 0$. When $a<0$ system (14) has three $2 \pi$-periodic solutions $\left(r^{i}(\theta, \varepsilon), \alpha^{i}(\theta, \varepsilon)\right)$ with $i=4,5,6$, such that $\left(r^{i}(\theta, \varepsilon), \alpha^{i}(\theta, \varepsilon)\right)$ tends to $\mathbf{x}_{i}$ when $\varepsilon \rightarrow 0$.

Now we analyze the stability of these periodic solutions from the eigenvalues of the Jacobian matrix $\mathcal{J}$ of $f_{2}(\mathbf{x})$ evaluated at $\mathbf{x}_{i}$ for $i=1, \ldots, 6$.

The eigenvalues of $\mathcal{J}$ evaluated at $(r, j \pi)$ are

$\lambda_{1,2}= \pm \pi /\left(\sqrt{10} \omega_{1}^{6}\right) \sqrt{m(r, a, h)}$

where

$$
\begin{aligned}
m(r, a, h)= & -\frac{10 a^{2} r^{2}\left(3 h^{2}-6 h r^{2}+2 r^{4}\right)}{r^{2}-2 h} \\
& -9 a\left(25 a^{2}+1\right)(-1)^{j} r^{3} \sqrt{2 h-r^{2}} .
\end{aligned}
$$

We are interested in the sign of $m(r, a, h)$ on the solutions of $f_{22}(r, j \pi)$. By proceeding as in Lemma 3 we see that there is no $(a, h)$ with $a \neq 0$ and $h>0$ such that $m(r, a, h)$ evaluated on the solutions of $f_{22}(r, j \pi)$ be 0 . In particular, we see that if $a>0$ then $\left.m(r, a, h)\right|_{\mathbf{x}_{1}}<0$, $\left.m(r, a, h)\right|_{\mathbf{x}_{2}}<0$ and $\left.m(r, a, h)\right|_{\mathbf{x}_{3}}>0$. If $a<0$ then $\left.m(r, a, h)\right|_{\mathbf{x}_{4}}<0,\left.m(r, a, h)\right|_{\mathbf{x}_{5}}>0$, and $\left.m(r, a, h)\right|_{\mathbf{x}_{6}}<0$. Hence the periodic solutions $\left(r^{i}(\theta, \varepsilon), \alpha^{i}(\theta, \varepsilon)\right)$ with $i=$ 3,5 are linearly unstable and the ones with $i=1,2,4,6$ are linearly stable. Clearly, the unstable orbits have a stable and an unstable manifolds formed by two cylinders.

Now we shall go back through the changes of variables in order to see how the $2 \pi$-periodic solutions $\left(r^{i}(\theta, \varepsilon)\right.$, $\left.\alpha^{i}(\theta, \varepsilon)\right)$, with $i=1, \ldots, 6$ of the differential system (14) looks in the initial Hamiltonian system (3). We substitute the solution $\left(r^{i}(\theta, \varepsilon), \alpha^{i}(\theta, \varepsilon)\right)$ into equation $H=h$ with $H$ given in (11) and we get $\rho^{i}(\theta, \varepsilon)$. Then

$\left(r^{i}(\theta, \varepsilon), \rho^{i}(\theta, \varepsilon), \alpha^{i}(\theta, \varepsilon)\right)$,

is a $2 \pi$-periodic solution for the differential system (12). This solution provides the $2 \pi / \omega_{1}$-periodic solution for the differential system (10)

$$
\begin{aligned}
& \left(r^{i}\left(\sigma^{i}, \varepsilon\right), \sigma^{i}, \rho^{i}\left(\sigma^{i}, \varepsilon\right), \alpha^{i}\left(\sigma^{i}, \varepsilon\right)\right)= \\
& \left.\left(r,-\omega_{1} t, \sqrt{2 h-r^{2}}, \alpha\right)\right|_{(r, \alpha)=\mathbf{x}^{i}}+O(\varepsilon),
\end{aligned}
$$

where $\sigma^{i}=\theta^{i}(t, \varepsilon)$. Going back to the change of variables (3) we get $2 \pi / \omega_{1}$ periodic solutions of system (3). Denoting $\mathcal{R}_{r_{i}}=\sqrt{2 h-r_{i}^{2}}$ and $\tau=t \omega_{1}$, their first order in $\varepsilon$ is: For $a>0$,

$$
\begin{aligned}
& \left(\frac{r_{2} \cos \tau}{\omega_{1}}, \frac{\mathcal{R}_{r_{2}} \cos (3 \tau)}{3 \omega_{1}},-r_{2} \sin \tau,-\mathcal{R}_{r_{2}} \sin (3 \tau)\right), \\
& \left(\frac{r_{1} \cos \tau}{\omega_{1}},-\frac{\mathcal{R}_{r_{1}} \cos (3 \tau)}{3 \omega_{1}},-r_{1} \sin \tau, \mathcal{R}_{r_{1}} \sin (3 \tau)\right), \\
& \left(\frac{r_{3} \cos \tau}{\omega_{1}},-\frac{\mathcal{R}_{r_{3}} \cos (3 \tau)}{3 \omega_{1}},-r_{3} \sin \tau, \mathcal{R}_{r_{3}} \sin (3 \tau)\right),
\end{aligned}
$$

and for $a<0$

$$
\begin{aligned}
& \left(\frac{r_{1} \cos \tau}{\omega_{1}}, \frac{\mathcal{R}_{r_{1}} \cos (3 \tau)}{3 \omega_{1}},-r_{1} \sin \tau,-\mathcal{R}_{r_{1}} \sin (3 \tau)\right), \\
& \left(\frac{r_{3} \cos \tau}{\omega_{1}}, \frac{\mathcal{R}_{r_{3}} \cos (3 \tau)}{3 \omega_{1}},-r_{3} \sin \tau,-\mathcal{R}_{r_{3}} \sin (3 \tau)\right), \\
& \left(\frac{r_{2} \cos \tau}{\omega_{1}},-\frac{\mathcal{R}_{r_{2}} \cos (3 \tau)}{3 \omega_{1}},-r_{2} \sin \tau, \mathcal{R}_{r_{2}} \sin (3 \tau)\right) .
\end{aligned}
$$

The first two periodic solutions in (17) are stable and the third one is unstable, whereas the first and the third periodic solutions in (18) are stable and the second one is unstable. Clearly these three solutions are different, so this completes the proof of statement (b) Theorem 1. 
Acknowledgements The first two authors are partially supported by MINECO grants number MTM2013-40998-P. The second author is also supported by an AGAUR grant number 2014SGR-568, and the grants FP7-PEOPLE-2012-IRSES 318999 and 316338. The third author is partially supported by FCT/Portugal through UID/ MAT/04459/2013.

\section{References}

Abramowitz, M., Stegun, I.A.: Handbook of Mathematical Functions with Formulas, Graphs, and Mathematical Tables, 9-th printing, p. 17. Dover, New York (1972).

Alfaro, F., Llibre, J., Pérez-Chavela, E.: A class of galactic potentials: periodic orbits and integrability. Astrophys. Space Sci. 344, 39 (2013)

Barbanis, B.: Escape regions of a quartic potential. Celest. Mech. Dyn. Astron. 48, 57 (1990)

Belmonte, C., Boccaletti, D., Pucacco, G.: On the orbit structure of the logarithmic potential. Astrophys. J. 669, 202 (2007)

Buică, A., Llibre, J.: Averaging methods for finding periodic orbits via Brouwer degree. Bull. Sci. Math. 128, 7 (2004)

Caranicolas, N.D.: A map for a group of resonant cases in quartic galactic Hamiltonian. J. Astrophys. Astron. 22, 309 (2001)

Caranicolas, N.D.: Orbits in global and local galactic potentials. Astron. Astrophys. Trans. 23, 241 (2004)

Caranicolas, N.D., Karanis, G.I.: Motion in a potential creating a weak bar structure. Astron. Astrophys. 342, 389 (1999)

Caranicolas, N.D., Innanen, K.A.: Periodic motion in perturbed elliptic oscillators. Astron. J. 103, 1308 (1992)

Caranicolas, N.D., Zotos, N.D.: Investigating the nature of motion in 3D perturbed elliptic oscillators displaying exact periodic orbits. Nonlinear Dyn. 69, 1795 (2012)

Contopoulos, G.: Orbits in highly perturbed dynamical systems. I. Periodic orbits. Astron. J. 75, 96 (1970a)

Contopoulos, G.: Orbits in highly perturbed dynamical systems. II. Stability of periodic orbits. Astron. J. 75, 108 (1970b)

Contopoulos, G., Moutsoulas, M.: Resonance cases and small divisors in a third integral of motion II. Astron. J. 70, 817 (1965)
Contopoulos, G., Zikides, M.: Periodic orbits and ergodic components of a resonant dynamical system. Astron. Astrophys. 90, 198 (1980)

Cushman, R.H., Dullin, H.R., Hanffmann, H., Schmidt, S.: The $1: \pm 2$ resonance. Regul. Chaotic Dyn. 12, 642 (2007)

Davoust, E.: Periodic orbits in a two-dimensional galactic potential. Celest. Mech. 31, 303 (1983)

Elipe, A., Miller, B., Vallejo, M.: Bifurcations in a non-symmetric cubic potential. Astron. Astrophys. 300, 722 (1995)

Giorgilli, A., Galgani, L.: From integrals from an autonomous Hamiltonian system near an equilibrium point. Celest. Mech. 17, 267 (1978)

Hénon, M., Heiles, C.: The applicability of the third integral of motion: some numerical experiments. Astron. J. 69, 73 (1964)

Karanis, G.I., Vozikis, L.C.: Fast detection of chaotic behavior in galactic potentials. Astron. Nachr. 329, 403 (2007)

Lang, S.: Algebra, 3rd. edn. Addison-Wesley, Reading (1993)

Lloyd, N.G.: Degree Theory. Cambridge Trends in Mathematics, vol. 73. Cambridge University Press, Cambridge (1978)

Marchesiello, A., Pucacco, G.: The symmetric $1: 2$ resonance. Eur. Phys. J. Plus 128(21), 14 (2013)

Miller, B.R.: The Lissajous transformation III. Parametric bifurcations. Celest. Mech. Dyn. Astron. 51, 251 (1991)

Olver, P.: Classical Invariant Theory. London Math. Soc. Student Texts, vol. 44. Cambridge University Press, New York (1999)

Pucacco, G., Marchesiello, A.: An energy-momentum map for the time-reversal symmetric $1: 1$ resonance with $\mathbb{Z}_{2} \times \mathbb{Z}_{2}$ symmetry. Physica D 271, 10 (2014)

Schmidt, S., Dullin, H.R.: Dynamics near the $p: \pm q$ resonance. Physica D 239, 1884 (2010)

Verhulst, F.: Nonlinear Differential Equations and Dynamical Systems. Springer, Berlin (1991)

Zotos, E.E.: Application of new dynamical spectra of orbits in Hamiltonian systems. Nonlinear Dyn. 69, 2041 (2012a)

Zotos, E.E.: The fast norm vector indicator (FNVI) method: a new dynamical parameter for detecting order and chaos in Hamiltonian systems. Nonlinear Dyn. 70, 951 (2012b) 